\title{
Le monde mouvant du cortex cérébral
}

Un numéro récent de $\mathrm{m} / \mathrm{s}$ a été consacré à la neuroplasticité, et notamment à la capacité que présentent certains circuits neuronaux adultes liés à des systèmes sensoriels ou moteurs à moduler leur activité en fonction de l'évolution des régions corporelles auxquelles ils sont liés ( $m / s n^{\circ} 9$, vol. 6, novembre 1990). Dans ce numéro, Robert Dykes avait notamment fait la synthèse des données suggérant que l'acétylcholine jouait un rôle dans la plasticité du cortex cérébral [1]. En renforçant ponctuellement l'activité de certains neurones au moment de l'arrivée d'un signal dans le circuit somatosensoriel cortical, l'acétylcholine pourrait autoriser la mise en route de mécanismes structuraux conduisant à une rétention de l'information, participant ainsi à des phénomènes de mémorisation au niveau cellulaire.

Sharon Juliano et al. [2] (USUHS, Washington DC, USA) démontrent aujourd'hui que l'acétylcholine participe également à un autre type de neuroplasticité des neurones du cortex somatosensoriel en modulant leur capacité à s'adapter à une situation nouvelle. Les travaux de l'équipe de Mike Merzenich de l'UCSF de San Francisco [3] ont démontré que le champ récepteur cutané à partir duquel on peut activer des neurones du cortex somatosensoriel s'étend lorsque l'on supprime expérimentalement celui auquel ils sont liés normalement. Ainsi, l'amputation d'un doigt entraîne l'activation des neurones correspondants par des stimulations somesthésiques appliquées sur les doigts voisins. Le rôle de l'acétylcholine dans ces phénomènes est apparemment majeur. Comme pour les mécanismes de mémorisation rapportés par Robert Dykes, la présence de ce neurotransmetteur semble indispensable à la réalisation du phé- trer, Juliano et al. ont utilisé une technique permettant le marquage de l'activité métabolique des neurones grâce à du 2-désoxy-D-glucose (2-DG) marqué au carbone 14. Les neurones sont de gros consommateurs de glucose et on peut rapporter cette consommation à leur activité métabolique en général. Comme l'activité métabolique neuronale est intimement liée à l'activité physiologique, toute modification ponctuelle de la consommation de glucose traduit un changement du fonctionnement des cellules. En quantifiant cette consommation, on pourrait ainsi établir des cartographies cérébrales $\mathrm{du}$ fonctionnement neuronal. Le glucose est, cependant, trop rapidement utilisé dans les chaînes métaboliques, et dégradé, pour être un marqueur utilisable de l'activité neuronale. La technique au 2-DG tire parti de l'incapacité dans laquelle se trouve la cellule nerveuse de métaboliser un faux sucre qu'elle capte dans le milieu extérieur comme s'il s'agissait de vrai glucose mais qui s'accumule ensuite dans la cellule sous forme de 2-DG-6-phosphate, sans dégradation évidente durant plusieurs dizaines de minutes. En injectant dans la circulation de faibles quantités de 2-DG marqué au ${ }^{14} \mathrm{C}$, on donne aux cellules nerveuses la possibilité de puiser pour leur fonctionnement un peu de ce marqueur au milieu de la quantité de glucose qui leur est nécessaire. On peut ainsi étudier l'effet de conditions expérimentales choisies (par exemple, dans l'étude citée, d'une stimulation somatosensorielle) sur le métabolisme neuronal en sacrifiant l'animal 45 minutes après injection et en réalisant une étude autoradiographique du marquage au ${ }^{14} \mathrm{C}$ sur les coupes de cerveau. La détermination de la consommation locale de glucose à partir du marquage du 2-DG s'effectue alors dans des conditions bien modélisées, notamment par Louis Sokoloff [4]. Grâce à cette technique au 2-DG, Juliano et al. ont vérifié les données obtenues par enregistrements électrophysiologiques par Merzenich. Ainsi, lorsque l'on ampute un doigt chez un rat adulte et que l'on applique une stimulation somesthésique sur un doigt adjacent (pendant le test au 2-DG), la région du cortex cérébral activée est beaucoup plus étendue que dans des conditions normales. Le rôle de l'acétylcholine dans cette plasticité est démontré dans la même étude par la comparaison entre les résultats obtenus chez des animaux témoins (amputés) et des animaux chez lesquels, en plus, on a détruit les neurones du cerveau antérieur qui fournissent normalement l'acétylcholine au cortex cérébral. Chez ces derniers, en effet, l'élargissement de la représentation corticale post-amputation n'apparaît pas.

L'acétylcholine paraît donc moduler l'efficacité des mécanismes qui contrôlent les modifications au long cours de l'activité neuronale et, en particulier, des représentations corticales sensorielles ou motrices. Plusieurs études indiquent que ces mécanismes eux-mêmes dépendent de circuits dans lesquels est impliqué l'acide $\boldsymbol{\gamma}$-amino-butyrique (GABA), neurotransmetteur inhibiteur quasi ubiquitaire du système nerveux central des mammifères. Utilisant la même technique au 2-DG, Juliano et al. [5] avait avancé cette hypothèse en observant que l'application de bicuculline (un antagoniste du GABA) sur la surface du cortex somatosensoriel d'un animal recevant une stimulation somesthétique provoquait un élargissement important de la région dans laquelle des neurones corticaux étaient activés. Jacobs et Donoghue [6] rapportent des résultats similaires à propos du cortex moteur 
chez le rat, obtenus sur la base d'enregistrements électrophysiologiques en présence ou en l'absence de bicuculline. Ces auteurs ont ainsi vérifié que le GABA est bien impliqué dans la délimitation précise des champs récepteurs observés puisque son blocage transitoire provoque l'élargissement de ces champs.

Les systèmes permettant l'expression des mécanismes de neuroplasticité dans le cortex cérébral sont donc progressivement mis en évidence. Les observations rapportées ici et celles présentées par Robert Dykes concourent à démontrer le rôle fondamental de l'acétylcholine comme modulateur, non pas directement de l'activité neuronale, mais de mécanismes d'adaptation de cette activité. Si l'on envisage la neuroplasticité comme un phénomène d'adaptation continu et indispensable du fonctionnement des neurones, ce qui est de plus en plus suggéré, l'acétylcholine apparaît donc ainsi comme un maître d'œuvre privilégié dont toute atteinte - au cours de la maladie d'Alzheimer, par exemple - se traduira par des dysfonctionnements majeurs et multiformes.

M. P.

1. Dykes RW. Acétylcholine et plasticité neuronale du cortex somatosensoriel. médecine/sciences $1990 ; 6: 870-5$.

2. Juliano SL, Ma W, Eslin D. Cholinergic depletion prevents expansion of topographic maps in somatosensory cortex. Proc Natl Acad Sci USA 1991; 88 : 780-4.

3. Merzenich M, Recanzone G, Jenkins WM, et al. Cortical representational plasticity. In Rakic P, Singer W, eds. Neurobiology of Neocortex. New York : Wiley, 1988 : 41-67.

4. Sokoloff L, Reivich M, Kennedy MH, et al. ${ }^{14} \mathrm{C}$ deoxyglucose method for the measurement of local cerebral glucose utilization theory, procedure, and normal values in the conscious and anesthetized albino rat. $J \mathrm{Neu}$ rochem 28, 1977 ; 28 : 897-916.

5. Juliano SL, Whitsel BL, Tommerdahl M, Cheema SS. Determinants of patchy metabolic labeling in the somatosensory cortex of cats : a possible role for intrinsic inhibitory circuitry. J Neurosci $1988 ; 9: 1-12$.

6. Jacobs KM, Donoghue JP. Reshaping the cortical motor map by unmasking laten intracortical connections. Science $1991 ; 251$ : 944-7.

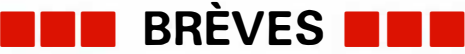

Régulation négative du récepteur CD4 par le gène nef du virus HIV. La protéine Nef du virus HIV jouerait un rôle régulateur négatif sur l'expression du génome viral, rôle encore controversé $(\mathrm{m} / \mathrm{s}$ $n^{\circ} 1$, vol. 7, p. 62). Une équipe de Seattle (WA, USA) [1] vient de montrer que l'hyperexpression du gène nef conduisait à une régulation négative de l'expression membranaire de la molécule CD4, récepteur principal des virus HIV. Un plasmide permettant l'hyperexpression de ce gène a été introduit dans les lymphocytes $\mathrm{T}, \mathrm{B}$ et des monocytes, exprimant tous la protéine CD4 à leur surface. L'accumulation de la protéine Nef dans les cellules transfectées s'accompagne d'une forte diminution de CD4 à la membrane cellulaire. Cette régulation négative ne se fait ni au niveau transcriptionnel (il n'y a pas de modification dans l'abondance de l'ARN messager codant pour CD4) ni au niveau traductionnel (la quantité totale de protéine CD4 reste inchangée). L'effet de la synthèse de Nef semble être d'empêcher le transport à la membrane de la protéine CD4, qui est accumulée dans le cytoplasme. Cet effet est indépendant de la phosphorylation de CD4, puisqu'un mutant non phosphorylable de cette protéine subit le même type de régulation par le gène nef. Cela signifie que la régulation négative de CD4 par nef n'emprunte pas la même voie que celle survenant après traitement par les esters de phorbol, des activateurs de la protéine kinase C. Dans ce dernier cas, en effet, la régulation dépend de la phosphorylation de CD4 et est abolie en cas de mutation des résidus sérine phosphorylables. Alors qu'on ne sait toujours pas si le gène nef est vraiment un régulateur négatif de l'expression du génome viral, ces résultats démontrent qu'il pourrait jouer un rôle dans la pathogénicité du virus, en diminuant l'expression de la protéine CD4, molécule d'adhérence jouant un rôle important dans la réponse immune. $\mathrm{La}$ disparition des cellules CD4, caractéristique des stades avancés du
SIDA, pourrait ainsi être secondaire, tout à la fois, à un processus de lyse cellulaire et à une inhibition de l'expression membranaire de la molécule CD4.

[1. Garcia VJ, Miller AD. Nature $1991 ; 350$ : 508-11.]

Création d'un rein de mammifère chimérique fonctionnel. Plusieurs études ont montré que des cellules infectées par des rétrovirus, surexprimant certains gènes spécifiques, pouvaient être intégrées dans divers organes. L'architecture du rein est très complexe, faite notamment d'interrelations entre des structures vasculaires et des néphrons. On pouvait penser que ce type d'intégration serait impossible. C'est néanmoins ce qu'ont réalisé A. S. Woolf et al. [1] (UCLA, USA, et University College, Londres, GB). Ils ont utilisé du rein embryonnaire, au stade de métanéphros, provenant soit de la lignée 83 de souris, transgénique (possédant de multiples copies du gène de la $\beta$-globine), soit de la lignée de souris beige dont beaucoup de cellules (parmi lesquelles les cellules tubulaires proximales rénales) contiennent des lysosomes géants facilement identifiables. Le fragment de métanéphros prélevé a été implanté dans le cortex rénal de souris nouveau-nées ; dans cette espèce, la néphrogenèse se prolonge dans la période post-natale. Le taux de survie des souris est de $50 \%$ et l'implantation n'est couronnée de succès que chez moins de $50 \%$ des survivants. L'intégration anatomique du métanéphros implanté est démontrée par hybridation in situ pour l'ADN de la $\beta$-globine ou par détection des lysosomes géants. Dans deux reins chimériques, les auteurs ont pu démontrer que du dextran marqué à la fluorescéine, injecté par voie intraveineuse se retrouvait dans les lumières de tubes proximaux, suggérant l'ultrafiltration du marqueur à travers les glomérules et la connexion entre des glomérules fonctionnels filtrants et des tubes proximaux.

[1. Woolf AS, et al. Kidney Int 1990; 38 : 991-7.] 